Fax : + 9101126588919.

E-mail: tanujdada@hotmail.com

Eye (2005) 19, 1347-1349. doi:10.1038/sj.eye.6701782; published online 24 December 2004

Sir,

\section{Greenish discoloration of a CeeON 911A silicone} intraocular lens

Since the advent by Charles Kelman in 1967, phacoemulsification has improved a lot. Foldable intraocular lens implants (IOL) are one of the most significant breakthrough after phacoemulsification. We here report a case of greenish discoloration of a silicone IOL (CeeOn 911A, Pharmacia Corporation). Exact cause of this discoloration is still unknown.

\section{Case report}

A 35-year-old woman underwent uneventful phacoemulsification with in the bag implantation of a +22.0 D silicon foldable IOL (CeeON 911A, Pharmacia Corporation, Serial No. 629507 211) under topical anaesthesia with $2 \%$ xylocain (Barrett- Hodgson, Pakistan) on 15 November 2001. No intraocular dye (trypan blue, indocyanine green) for staining of anterior capsule was used. Her postoperative recovery and follow-ups were unremarkable and at her last visit on $31 / 01 / 2002$, her visual acuity (VA) in her operated eye was 1.0 with -1.00 DS. She then lost to follow-up.

On 10 March 2004, she presented with complaint of decreasing vision in her left (other) eye. Examination revealed cataract in her left eye, resulting in decreased VA. Interestingly her right eye, which was operated for cataract in 2001, caught more attention. The IOL in the right eye was positioned in the bag, well placed and well centred with anterior capsular opacification. There was no posterior capsular opacity, but one-third of both the anterior and the posterior of the IOL were green in colour. (Figure 1). The centre was clear and so were the haptics. VA of the patient in this particular eye was same as recorded earlier in 2002, that is, 1.0 with -1.0 DS. Contrast sensitivity was not carried out. Captivatingly, the patient was unaware of the change in colour of IOL and there were no visual complaints.

In order to determine the cause of discoloration of IOL, she was inquired comprehensively. She was asked questions pertaining to the use of medication, systemic illness, and any invasive procedure especially indocyanine green angiography or fundus fluorescein angiography during the preceding years. Nothing

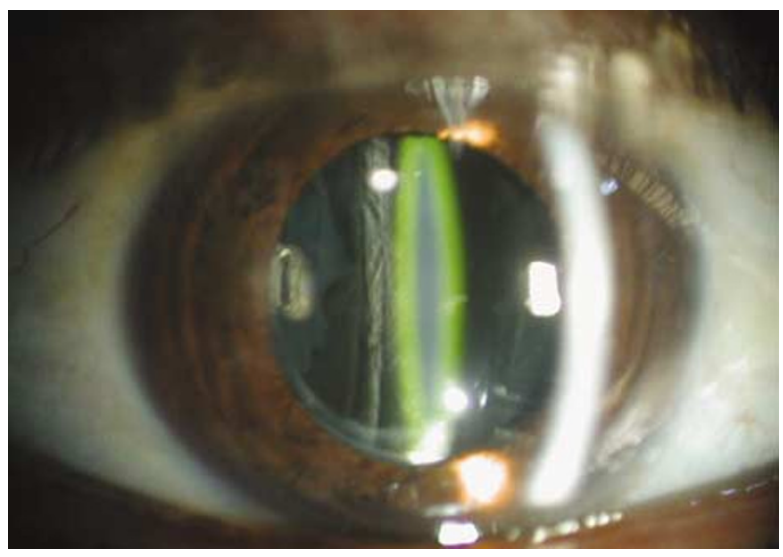

Figure 1 Slitlamp photograph of greenish discoloration of CeeOn 911A IOL.

affirmative was reported, which could be suspected as a cause for greenish colour of IOL.

\section{Discussion}

Even when the most common intraocular surgery is performed uneventfully, something unexpected can jump up into the scene. The case cited above is a unique example of 'an extraordinary development in an ordinary treatment'

Foldable IOLs are in use since mid-1980s. In this particular case, CeeOn 911A was implanted in the patient's eye that turned green in almost 3 years. CeeOn 911A is a UV-absorbing foldable lens having silicone optic and haptics made of polyvinylidene fluoride homopolymer (PVDF). Although large case series of silicone IOL implantation has demonstrated excellent visual outcomes, ${ }^{1,2}$ various ophthalmologists reported cases of adverse events with silicone lenses. To the very best of our knowledge, no case of greenish discoloration of CeeOn lens has ever been reported earlier.

In 1991, Milauskas ${ }^{3}$ reported brownish discoloration of varying intensity of silicone IOLs (manufactured by STAAR Surgical and IOLAB Corporation) in a series of 15 cases, 4-5 years after surgery. VA of all the patients under observation remained stable. At that time, Milauskas questioned the long-term stability of silicone IOLs in human eyes. Koch and Heit ${ }^{4}$ in 1991 reported two cases of brownish discoloration of silicone IOLs. However, use of silicone IOLs in human eyes continues till today. Manufacturers claim use of newer and improved generation silicone material in these lenses. However, recently, Tanaka et $a l^{5}$ reported brown haze in an Allergan SI-40NB silicone IOL on the very next day of surgery.

Exact causative factor has not yet been identified; few speculative mechanisms can, however, be addressed. 
Firstly, all the discolorations previously published were brown, mostly from USA, Japan, and Europe. In contrast, we report a greenish discoloration, from Asian subcontinent where weather is extreme in nature. It is too hot, humid with bright sunshine in summer and too cold in winter. Socially, the housewives spend much time in kitchens where exposure to heat is immense and inevitable. The exposure to these extreme temperatures may cause change in colour of the IOL. A manufacturing defect cannot be ruled out nor can the inherent problem with the silicone material used, maybe with some impurities.

We yet do not know how much the IOL will further gain greenish colour or deteriorate the vision of the patient. In both the extreme situations, the affected IOL has to be replaced with a newer one. The analysis of explanted IOL (if removed at any time in future) may be helpful in determining the exact cause of this green discoloration. Till then, the question rose by Milauskas in 1991 regarding implantation of silicone IOLs in human beings holds ground and the safety and efficacy of their use need reassessment.

\section{References}

1 Lemagne JM. Results of the intraocular lens SI-30 implantation. Bull Soc Belge Ophthalmol 1996; 262: 155-157.

2 Steinert RF, Bayliss B, Brint SF, Giamporcaro JE, Hunkeler JD. Long term clinical results of AMO Phacoflex model SI-18 intraocular lens implantation. J Cataract Refract Surg. 1995; 21(3): 331-338.

3 Milauskas AT. Silicone intraocular lens implant discoloration in humans. Arch Ophthalmol 1991; 109: 913-916.

4 Koch DD, Heit LE. Discoloration of silicone intraocular lenses. Arch Ophthalmol 1991; 110: 319-320.

5 Tanaka T, Saika S, Hashizume N, Ohnishi Y. Brown haze in an Allergan SI-40NB silicone intraocular lens. J Cataract Refract Surg. 2004; 30: 250-252.

M Siddique1, KM Ashraf ${ }^{1}$ and ZA Qazi

${ }^{1}$ Department of Ophthalmology, Laser Vision

Center, 95K, Model Town, Lahore, Pakistan

${ }^{2}$ LRBT Eye Hospital, Lahore, Pakistan

Correspondence: M Siddique,

Tel: + 9242 5868844;

Fax: + 9242 5839968,

E-mail: mustansirs@hotmail.com

Eye (2005) 19, 1349-1350. doi:10.1038/sj.eye.6701783; published online 28 January 2005
Sir,

Concurrent $v s$ dependent retinal arteriolar occlusion and central vein occlusion

Ozdek et $a l^{1}$ report details of two patients with hyperhomocysteinaemia who presented with nonischaemic occlusion of the central retinal vein (CRV) and putative concurrent occlusion of a branch retinal arteriole. They make reference to an earlier description of seven eyes similarly affected by CRV occlusion and segmental inner retinal infarction ${ }^{2}$ in which cilioretinal infarction, secondary to the CRV occlusion, ${ }^{3,4}$ was discounted as the mechanism of ischaemia. However, a single vascular luminal obstruction is more likely than two simultaneous (but separate) occlusions, especially in young patients. The possibility of cilioretinal infarction should be seriously entertained, therefore, even if this implies that up to half of the retinal circulation must perforce derive from posterior ciliary branches of the ophthalmic artery. Indeed, in Case 1 of this latest report, ${ }^{1}$ the inferotemporal vessel supplying the territory of the infarct appears to have a 'hook' characteristic of a cilioretinal arteriole as it emerges from the disc rim.

In CRV occlusions, the distinction between a (simultaneous) branch arteriolar occlusion and a (consequential) cilioretinal arteriolar occlusion is not merely of academic interest. Recognition of the dependent association will spare the patient from unnecessary investigations, including the search for a source of arteriolar embolism. Moreover, therapeutic lowering of the intraocular pressure (as attempted in Case 1) runs the risk of precipitating retinal haemorrhage by increasing transmural hydrostatic pressure gradients, without any prospect of improving inner retinal perfusion. ${ }^{5}$

\section{References}

1 Osdek S, Yulek F, Gurelik G et al. Simultaneous central retinal vein and retinal artery branch occlusions in two patients with homocystinaemia. Eye 2004; 18: 942-945.

2 Duker JS, Cohen MS, Brown GC et al. Combined branch retinal artery and central retinal vein obstruction. Retina 1990; 10: $105-112$.

3 McLeod D, Ring CP. Cilio-retinal infarction after retinal vein occlusion. Br J Ophthalmol 1976; 60: 419-427.

4 McLeod D. Central retinal vein obstruction with cilio-retinal infarction. Eye 2003; 17: 283.

5 McLeod D. Cilio-retinal arterial circulation in central retinal vein occlusion. Br J Ophthalmol 1975; 59: 486-492.

\section{McLeod}

Academic Dept of Ophthalmology, Manchester

Royal Eye Hospital, Oxford Road, Manchester M13

9WH, UK 